\title{
Antipsychotic Drug Use and Screening for Delirium in Mechanically Ventilated Patients in Canadian Intensive Care Units: An Observational Study
}

\author{
Zoé Thiboutot, Marc M Perreault, David R Williamson, Louise Rose, Sangeeta Mehta, \\ Melanie D Guenette, Deborah Cook, and Lisa Burry
}

\begin{abstract}
Background: Critically ill patients frequently experience delirium, and antipsychotic drugs are often used to manage symptoms.

Objectives: To describe the use of antipsychotic drugs and delirium screening tools in mechanically ventilated, critically ill adult patients in Canadian intensive care units (ICUs) and to identify factors associated with the use of antipsychotic drugs.

Methods: Pharmacists from 51 Canadian ICUs prospectively collected data on antipsychotic use and delirium screening in all patients for whom invasive mechanical ventilation was initiated during a chosen 2 -week period occurring sometime in 2008 or 2009.

Results: Data were collected for a total of 712 patients, of whom 115 $(16.2 \%)$ received at least one dose of an antipsychotic. The antipsychotic prescribed, the total daily dose, and the administration schedule varied across sites. Delirium screening tools, validated for use in mechanically ventilated patients and endorsed by professional society guidelines, were part of routine care in a minority of ICUs (7/51 [13.7\%]), and delirium screening was documented for few patients overall (41/712 patients [5.8\%]). In a multivariable analysis, administration of antipsychotics was independently associated with longer duration of mechanical ventilation (odds ratio [OR] 1.12, 95\% confidence interval [CI] 1.07-1.17), daily interruption of sedation (OR 1.71, 95\% CI 1.01-2.90), and use of physical restraints (OR 2.15, 95\% CI 1.27-3.65).

Conclusion: A minority of mechanically ventilated patients in Canadian ICUs received antipsychotic drugs, and screening for delirium with validated tools was rare. Antipsychotic drug use was independently associated with longer duration of mechanical ventilation, daily interruption of sedation, and use of physical restraints.
\end{abstract}

Keywords: antipsychotic, delirium, screening, mechanical ventilation, intensive care
Can J Hosp Pharm. 2016;69(2):107-13

\section{RÉSUMÉ}

Contexte : Les patients gravement malades souffrent fréquemment de délire, une affection dont les symptômes sont souvent traités à l'aide d'antipsychotiques.

Objectifs : Dresser le portrait de l'utilisation des antipsychotiques et des outils de dépistage du délire chez les patients adultes gravement malades sous ventilation mécanique ayant séjourné dans une unité de soins intensifs (USI) canadienne et relever les facteurs associés à l'utilisation des antipsychotiques.

Méthodes : Des pharmaciens de 51 USI canadiennes ont recueilli des données de façon prospective sur l'utilisation des antipsychotiques et sur le dépistage de cas de délire auprès de tous les patients placés sous ventilation mécanique effractive au cours d'une période de deux semaines entre 2008 et 2009.

Résultats : Les données ont été recueillies chez un total de 712 patients. De ce nombre, 115 (16,2 \%) ont reçu au moins une dose d'un antipsychotique. L'antipsychotique prescrit, la posologie quotidienne et l'horaire d'administration variaient d'un établissement à l'autre. Des outils de dépistage du délire (dont l'emploi est validé chez les patients sous ventilation mécanique et approuvé dans les lignes directrices d'associations professionnelles) ne faisaient partie des soins habituels que dans un petit nombre d'USI (7/51 [13,7\%]). De plus, les dépistages du délire n’ont été consignés que pour peu de patients dans l'ensemble (41/712 patients $[5,8 \%])$. Au cours d'une analyse multivariable, on a associé indépendamment l'administration d'antipsychotiques à une durée accrue de la ventilation mécanique (risque relatif approché [RRA] de 1,12, intervalle de confiance [IC] à $95 \%$ de 1,07-1,17), à une interruption quotidienne de la sédation (RRA de 1,71 , IC à $95 \%$ de $1,01-2,90$ ) et à l'emploi de contention physique (RRA de 2,15, IC à $95 \%$ de 1,27-3,65).

Conclusions : Seul un petit nombre de patients sous ventilation mécanique ayant séjourné dans une USI canadienne ont reçu des antipsychotiques; de plus, des outils validés de dépistage du délire n’ont que rarement été employés. L'utilisation d'antipsychotiques a été indépendamment associée à une durée accrue de la ventilation mécanique, à l'interruption quotidienne de la sédation et à l'emploi de contention physique.

Mots clés : antipsychotique, délire, dépistage, ventilation mécanique, soins intensifs 


\section{INTRODUCTION}

$\mathrm{D}$ elirium, an acute confusional state, is associated with adverse clinical outcomes in critically ill patients. ${ }^{1-6}$ Professional society guidelines ${ }^{7,8}$ recommend the routine use of screening tools specifically designed for detecting delirium in critically ill patients (e.g., the Confusion Assessment Method for the Intensive Care Unit [CAM-ICU] ${ }^{9}$ and the Intensive Care Delirium Screening Checklist $\left.[\mathrm{ICDSC}]^{10}\right)$. However, international surveys and observational studies have indicated that delirium screening tools are not widely incorporated into critical care practice, ${ }^{11-16}$ which represents a missed opportunity to detect the syndrome. As such, delirium may go unrecognized.

The guidelines of the Society of Critical Care Medicine ${ }^{7,8}$ suggest that when delirium is detected, reversible causes be addressed first (e.g., by treating underlying infection, reorienting patients, or modifying drug regimens), given that effective pharmacological treatment has yet to be identified. However, international surveys and observational studies indicate that antipsychotic drugs are commonly used in the context of critical care, ${ }^{12-16}$ including in the management of delirium (e.g., psychomotor agitation), ${ }^{12,13}$ despite the absence of evidence showing improved clinical outcomes with their use.

A previously reported multicentre, prospective study was undertaken to describe the use of sedatives, opioids, antipsychotics, and neuromuscular blocking agents in 51 Canadian intensive care units (ICUs). ${ }^{17}$ The current article reports a planned analysis of the study database, which had the primary objective of describing the use of antipsychotic drugs and delirium screening tools in critically ill, mechanically ventilated adults. The secondary objective was to identify factors associated with antipsychotic drug use in this study cohort.

\section{METHODS}

\section{Study Design}

The original study, for which the current article reports a subanalysis, was a national prospective audit of practice investigating the use of sedatives, analgesics, antipsychotics, and neuromuscular blockers in critically ill adult patients who were receiving mechanical ventilation. ${ }^{17}$ Details of the study methods have been previously published in full. ${ }^{17,18}$ Data were prospectively collected by pharmacists practising in 51 ICUs in both university-affiliated and community hospitals across all 10 Canadian provinces. Study approval was obtained from each local institutional review board, and the need for informed consent was waived at each site.

\section{Patient Population}

Patients 16 years of age or older with invasive mechanical ventilation initiated during a site-specific 2 -week study period in
2008 or 2009 were consecutively enrolled. Data were collected from initiation of mechanical ventilation until one of the following end points: $24 \mathrm{~h}$ after extubation or tracheostomy, death, or 30 th day of mechanical ventilation.

\section{Data Collection}

A standardized case report form was used to collect the defined data for each institution (e.g., community or universityaffiliated hospital), ICU (open or closed ICU; bed count; implementation of assessment tools and protocols for sedation, analgesia, delirium, and chemical paralysis), and patient. Patient data included demographic characteristics (e.g., age, sex); Acute Physiology and Chronic Health Evaluation (APACHE) II score; past medical and medication history; and history of smoking, alcohol use, and/or illicit drug use. The following data were collected on each study day: all sedative, analgesic, antipsychotic, and neuromuscular blocker use, including dose, frequency, and route of administration; whether use of these drugs was guided by standardized protocols; use of assessment tools and/or specific protocols for sedation, analgesia, and delirium management; use of physical restraints; and adverse events, specifically inadvertent device removal or patient behaviour suggestive of being a danger to self or to others.

\section{Statistical Analyses}

Antipsychotic use was defined as the administration of at least one dose of an antipsychotic drug during the study period. Demographic characteristics, clinical variables, and details of antipsychotic drug use are reported as means (with standard deviation [SD] and range) or as counts and proportions, as appropriate.

A multivariable logistic regression model was generated to evaluate the independent effects of various demographic and clinical factors on antipsychotic drug use. The following variables for entry into the model were selected a priori, on the basis of published studies and clinical importance $e^{6,8,19-21}$ : age, APACHE II score, use of antipsychotic before ICU admission (a potential marker of delirium before transfer to the ICU), duration of mechanical ventilation, exposure to benzodiazepines (expressed in midazolam equivalents), agitation (defined as a score $>4$ on the Riker Sedation-Agitation Scale [SAS]), ${ }^{22}$ use of daily sedation interruption, use of a delirium screening tool, and application of physical restraints. Patients with a documented history of schizophrenia who were treated with antipsychotics were excluded from the multivariable analysis. Results are reported as odds ratios (ORs) with 95\% confidence intervals (CIs). Before the multivariable modelling, variables were assessed for multicollinearity using tolerance statistics. A tolerance value less than 0.4 was defined as indicating the presence of multicollinearity. In such cases, only one member of a correlated set was to be included in the multivariable model; however, multicollinearity 
was not found to be a concern for the selected variables of interest. The maximum number of variables retained in the model was based on rules of modelling for logistic regression. ${ }^{23}$ All tests were 2 -tailed, with a $p$ value of 0.05 or below being deemed significant. An independent statistician conducted all analyses using SAS 9.2 software (SAS Institute, Cary, North Carolina).

\section{RESULTS}

\section{Baseline Patient and Hospital Characteristics}

Data were collected for a total of 712 patients. The mean age was 60.8 years (SD 16.7 years), 443 (62.2\%) were male, and the mean APACHE II score was 19.6 (SD 7.9) (Table 1). Further details on hospital and baseline patient characteristics have been previously reported. ${ }^{17,18} \mathrm{~A}$ delirium assessment tool was implemented as part of routine ICU practice at 7 (13.7\%) of the 51 sites: 4 sites used the CAM-ICU, ${ }^{9} 2$ sites used the ICDSC, ${ }^{10}$ and 1 site used the NEECHAM confusion scale. ${ }^{24}$

\section{Antipsychotic Drug Use}

No site used a protocol to manage prescribing of antipsychotics. An antipsychotic drug was administered at least once to $115(16.2 \%)$ of the 712 patients enrolled in the study. Of the
115 patients who received an antipsychotic while in the ICU, 22 (19.1\%) had received an antipsychotic at home for a documented diagnosis of schizophrenia, and 34 (29.6\%) were given an antipsychotic in hospital before the ICU admission (e.g., for sleep or to treat agitation). An antipsychotic drug was administered on 1009 (27.9\%) of 3620 ICU study days.

Twenty of the patients were treated with 2 or more antipsychotics, for a total of 143 prescriptions. Both typical and atypical antipsychotic agents were prescribed. Of the 115 patients who received antipsychotic agents, 44 (38.3\%) received the drug solely as an "as needed" therapy (Table 2); the remainder received fixed-interval dosing, either exclusively (39 [33.9\%]) or in combination with "as-needed" therapy (32 [27.8\%]). Intravenous haloperidol was the most commonly prescribed antipsychotic, followed by enteral atypical agents (Table 2). There was a wide range in administered doses for all antipsychotics used. Antipsychotics were initiated on average 4.8 days (SD 4.3 days) after admission to the ICU and were given for an average of 4.1 days (SD 4.2 days).

\section{Screening for Delirium}

Screening for delirium was rarely performed (41 [5.8\%] of the 712 patients; 148 [4.1\%] of the 3620 total ICU days),

\section{Table 1. Baseline Characteristics of Patients in a Study of Antipsychotic Use in Canadian ICUs}

\begin{tabular}{|c|c|c|}
\hline Characteristic & \multicolumn{2}{|c|}{$\begin{array}{c}\text { No. (\%) of Patients* } \\
(n=712) \dagger\end{array}$} \\
\hline Age (years), mean \pm SD & \multicolumn{2}{|c|}{$60.8 \pm 16.7$} \\
\hline Sex, male & 443 & $(62.2)$ \\
\hline University hospital & 497 & $(69.8)$ \\
\hline APACHE II score, mean \pm SD & \multicolumn{2}{|c|}{$19.6 \pm 7.9$} \\
\hline \multicolumn{3}{|l|}{ Admission type } \\
\hline Medical & 280 & (39.3) \\
\hline Surgical & 248 & $(34.8)$ \\
\hline Cardiac & 85 & (11.9) \\
\hline Trauma & 35 & $(4.9)$ \\
\hline Other $\neq$ & 64 & $(9.0)$ \\
\hline \multicolumn{3}{|l|}{ Medical history } \\
\hline Neurological disease§ & 92 & $(12.9)$ \\
\hline Psychiatric diseaseף & 98 & (13.8) \\
\hline \multicolumn{3}{|c|}{ Medication history or social habits before ICU admission } \\
\hline Antipsychotics & 56 & $(7.9)$ \\
\hline Tobacco** & 125 & $(17.6)$ \\
\hline Alcoholt† & 181 & $(25.4)$ \\
\hline Recreational drug use & 33 & $(4.6)$ \\
\hline \multicolumn{3}{|c|}{ 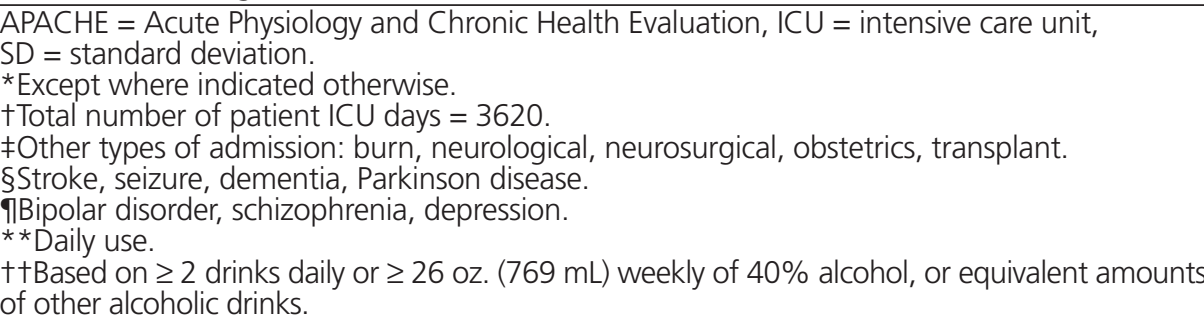 } \\
\hline
\end{tabular}


This single copy is for your personal, non-commercial use only.

For permission to reprint multiple copies or to order presentation-ready copies for distribution, contact CJHP at cjhpedit@cshp.ca

\section{Table 2. Characteristics of Antipsychotic Therapy in Canadian ICUs}

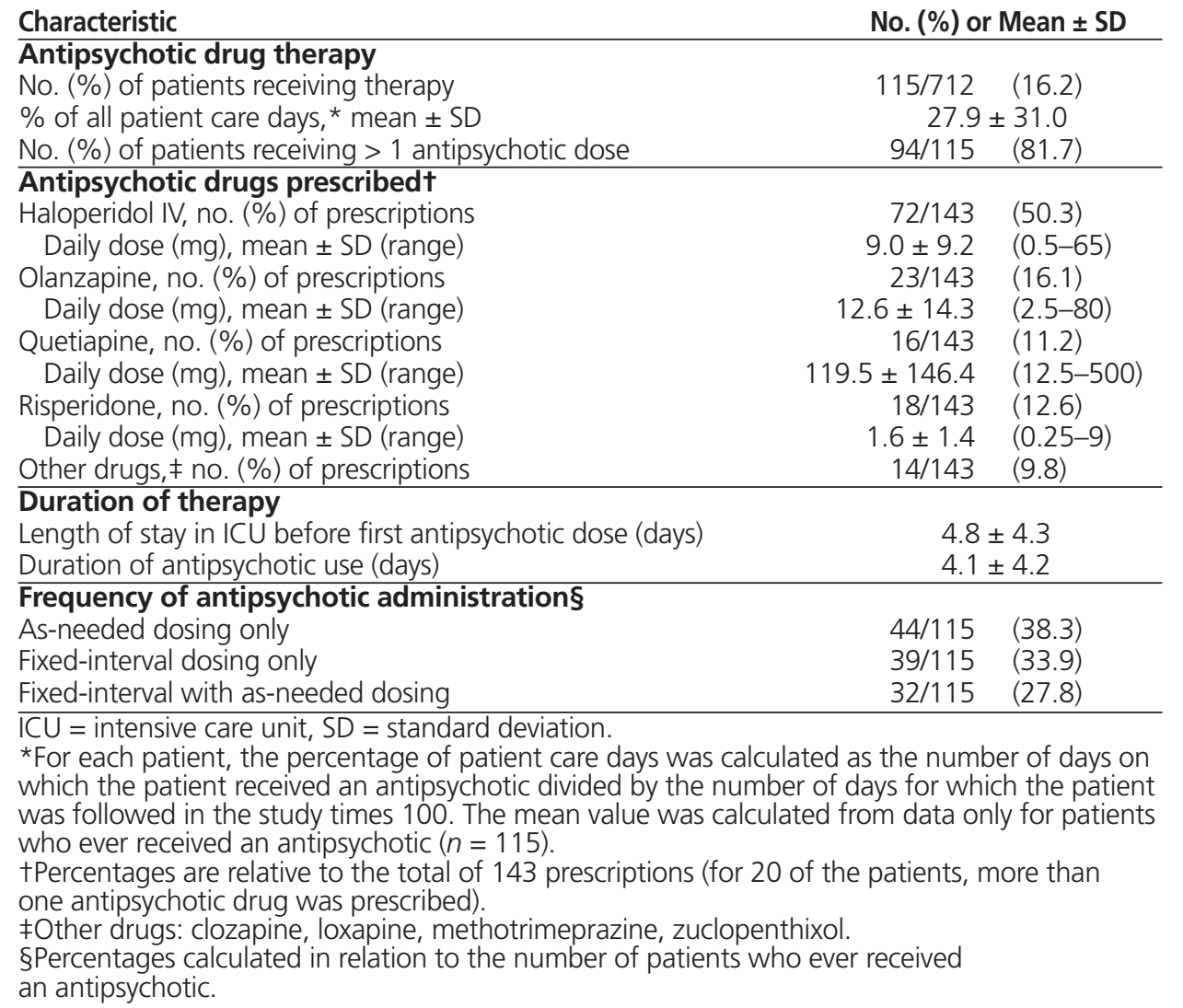

irrespective of hospital policy regarding screening (Table 3). Delirium screening was more common in ICUs where use of an assessment tool was implemented as part of standard ICU practice. In the 7 ICUs where an assessment tool was incorporated into standard ICU practice, 36 (17.1\%) of the 210 patients were screened for delirium at least once; in the 44 ICUs that did not use such tools as part of standard ICU practice, screening was less common, occurring at least once in only $8(1.6 \%)$ of 502 patients $(p<0.001)$. There was no difference in the use of antipsychotics between ICUs with and without delirium screening incorporated into routine practice (33 [15.7\%] of 210 patients, for a mean duration of 4.5 days, versus 82 [16.3\%] of 502 patients, for a mean duration of 3.8 days; $p=0.62$ ).

\section{Factors Associated with Antipsychotic Drug Use}

In the multivariable analysis (Table 4), longer duration of mechanical ventilation (OR 1.12, 95\% CI 1.07-1.17; $p<0.001$ ), daily sedation interruption (OR 1.71, 95\% CI 1.01-2.90; $p=0.05$ ), and use of physical restraints (OR 2.15, 95\% CI $1.27-3.65 ; p=0.004)$ were independently associated with receipt of an antipsychotic drug when adjusted for age, APACHE II score at ICU admission, prior use of antipsychotic, total benzodiazepine exposure (expressed in midazolam equivalents), agitation (Riker SAS score $>4$ ), and delirium screening.

\section{DISCUSSION}

This multicentre observational study showed that delirium screening tools validated for use in mechanically ventilated patients were implemented as a standard of care in a minority of Canadian ICUs (13.7\%). Screening was more frequent at sites where such tools were incorporated into standard practice than at sites not using the tools consistently $(17.1 \%$ versus $1.6 \%$ of patients screened at least once), yet overall delirium screening was rare $(5.8 \%$ of patients). Consequently, it is reasonable to posit that delirium is likely under-recognized in Canadian ICUs. At least one instance of antipsychotic drug use was identified in $16.2 \%$ of the patients, on $27.9 \%$ of ICU days. Prescribing patterns were variable, however, and were not associated with use of delirium screening tools as part of standard ICU practice. Antipsychotic drug use was independently associated with a longer duration of mechanical ventilation, daily sedation interruption, and use of physical restraints. We suggest caution in inferring causality, as the direction of interaction between the use of antipsychotics and duration of mechanical ventilation, for example, remains unclear.

Given the substantial morbidity and mortality associated with delirium in critically ill patients, current guidelines recommend routine patient screening with validated assessment tools, vigilance with regard to potential precipitating and predisposing 


\section{Table 3. Delirium Screening in Canadian ICUs}

\begin{tabular}{|c|c|c|c|}
\hline \multicolumn{4}{|l|}{ Variable } \\
\hline \multicolumn{2}{|c|}{$\begin{array}{l}\text { No. }(\%) \text { of ICUs with delirium assessment tool implemented } \\
\text { in routine practice }\end{array}$} & 51 & $(13.7)$ \\
\hline \multicolumn{4}{|c|}{ No. $(\%)$ of ICUs using various assessment tools } \\
\hline CAM-ICU ${ }^{9}$ & \multicolumn{2}{|c|}{$4 / 51$} & (7.8) \\
\hline ICDSC $^{10}$ & \multicolumn{2}{|c|}{$2 / 51$} & (3.9) \\
\hline NEECHAM confusion scale ${ }^{24}$ & \multicolumn{2}{|c|}{$1 / 51$} & $(2.0)$ \\
\hline \multicolumn{4}{|l|}{ Delirium screening } \\
\hline No. $(\%)$ of patients with screening & \multicolumn{2}{|c|}{$41 / 712$} & $(5.8)$ \\
\hline No. (\%) of ICU days with screening & \multicolumn{2}{|c|}{$148 / 3620$} & $(4.1)$ \\
\hline \multicolumn{4}{|l|}{ No. $(\%)$ of assessments performed* } \\
\hline By ICU nurse & \multicolumn{2}{|c|}{$89 / 148$} & $(60.1)$ \\
\hline By physician trainee & \multicolumn{2}{|c|}{$39 / 148$} & $(26.4)$ \\
\hline By ICU attending physician & \multicolumn{2}{|c|}{$19 / 148$} & $(12.8)$ \\
\hline By psychiatrist & \multicolumn{2}{|c|}{$1 / 148$} & $(0.7)$ \\
\hline \multicolumn{4}{|c|}{$\begin{array}{l}\text { CAM-ICU = Confusion Assessment Method for the Intensive Care Unit, } \\
\text { ICDSC = Intensive Care Delirium Screening Checklist, ICU = intensive care unit. } \\
\text { *Based on } 148 \text { assessments. }\end{array}$} \\
\hline \multicolumn{4}{|c|}{$\begin{array}{l}\text { Table 4. Multivariable Analysis of Factors Associated with Antipsychotic Use } \\
\text { in Canadian ICUs }\end{array}$} \\
\hline & \multicolumn{3}{|c|}{ Type of Analysis; OR (95\% Wald CI) } \\
\hline Variable & Univariable & & ultivariable \\
\hline Age & $1.00(1.00-1.01)$ & 1.0 & $(0.98-1.01)$ \\
\hline APACHE II score & $1.02(0.99-1.05)$ & 1.0 & $2(0.99-1.05)$ \\
\hline Use of antipsychotic before ICU admission* & $1.27(0.57-2.83)$ & 1.1 & $(0.45-2.72)$ \\
\hline Days of mechanical ventilation & $1.17(1.13-1.22)$ & & $2(1.07-1.17)$ \\
\hline Total dose of midazolam equivalents $†$ & $1.00(1.00-1.00)$ & & $(1.00-1.00)$ \\
\hline Any agitation (Riker SAS score $>4)^{22}$ & $3.67(2.15-6.27)$ & 1.7 & $3(0.95-3.33)$ \\
\hline Any daily interruption of sedation & $2.77(1.73-4.46)$ & 1.7 & $(1.01-2.90)$ \\
\hline Any screening for delirium & $3.54(1.83-6.84)$ & 2.0 & $5(0.92-4.58)$ \\
\hline Any restraint use & $4.03(2.49-6.51)$ & 2.1 & $(1.27-3.65)$ \\
\hline
\end{tabular}

factors, and implementation of nonpharmacological prevention and treatment approaches, such as early mobilization. ${ }^{8}$ Efficacy and safety data on the use of antipsychotics in critically ill patients are lacking. The role of such drugs for treatment of delirium in particular awaits the results of adequately powered interventional trials (e.g., the MIND-USA trial, clinicaltrials.gov NCT01211522), especially given that existing data on important clinical outcomes are conflicting. Randomized controlled trials have not shown haloperidol to alter delirium-related outcomes of critically ill patients ${ }^{25,26}$; atypical antipsychotics were shown to reduce the duration of delirium in one pilot study. ${ }^{27}$

In the current study, antipsychotic drugs were used relatively late in patients' ICU admission, when it is most likely that medical stabilization had taken place and attempts were being made to wean patients from sedation and mechanical ventilation. However, because objective delirium screening was infrequent, we can only speculate on the reasons for the use of antipsychotic drugs. These drugs may have been administered for delirium, to manage psychomotor agitation, to complement the use of physical restraints, or for sedation or sleep (i.e., as an alternative to traditional sedatives). Because antipsychotic drug use was associated with daily sedation interruption, it is possible that such lightened sedation may have revealed agitation and/or delirium. This hypothesis is supported by the finding that antipsychotic drug use was also associated with the use of physical restraints, which should, in theory, not be required for sedated or nonagitated patients. ${ }^{18}$

Low adoption of delirium assessment tools has been reported in several other studies in various countries. The results reported here are similar to those obtained in a retrospective cohort study conducted at 71 US academic hospitals in 2010.13 In that study, delirium was diagnosed in only $6 \%$ of hospitalizations with an ICU admission, and antipsychotics were administered to $11 \%$ of patients. Haloperidol was most commonly prescribed (62\% of patients), followed by quetiapine $(31 \%){ }^{13}$ In a survey investigating sedation and delirium management practices in 23 ICUs in Australia and New Zealand, ${ }^{28}$ only 2 sites (9\%) reported routine use of a delirium scale. Of the 234 patients, only 56 were assessed for delirium, with $12(21 \%)$ of these screening positive. In a survey of 1015 Brazilian ICU physicians, ${ }^{29}$ only $13 \%$ reported using a validated screening tool 
for delirium, and more than $90 \%$ cited "clinical evaluation" as a method of diagnosis. ${ }^{29}$ However, $38 \%$ reported not screening for delirium whatsoever, while the remainder reported screening once or more per day. When asked how delirium was managed, $88 \%$ of respondents said that haloperidol was used, and $45 \%$ and 36\% used benzodiazepines and atypical antipsychotics, respectively (multiple answers were permitted). In a 2-part study (survey and observational study) of 53 ICU pharmacists in the United States, ${ }^{15} 25 \%$ of respondents reported that delirium was routinely assessed, but only $10 \%$ of patients underwent assessment during the study period. Most of the survey respondents $(88 \%)$ selected haloperidol as the preferred drug for delirium. Within the observational study, a small percentage of all patients ( $5 \%$ and $6 \%$, respectively) received a scheduled or an as-needed antipsychotic medication during the study period; haloperidol was the most frequently prescribed antipsychotic overall, primarily because of its use on as as-needed basis (accounting for $89 \%$ of as-needed antipsychotic doses). ${ }^{15}$

The current study is, to the authors' knowledge, the first prospective description of Canadian ICU practices regarding antipsychotic drug use and delirium screening in mechanically ventilated patients, where delirium rates have been reported to be as high as $40 \%-80 \% .{ }^{8}$ The strengths of this study included prospective data collection, large sample size, multicentre design, and multiprovince representation. Nonetheless, the study had limitations. Neither the indication for antipsychotic use nor the use of nonpharmacological strategies for the prevention and treatment of delirium was recorded. For each patient who reached the end point of extubation or tracheostomy, data collection stopped 1 day after the end point; as such, the actual percentage of patients treated with antipsychotics may have been higher than reported here, and the overall duration of use longer, if therapy was continued after ICU discharge. Jasiak and others ${ }^{30}$ reported that antipsychotics prescribed for ICU delirium are often continued after discharge from the ICU or from hospital, and long-term use may be associated with adverse effects and unnecessary health care costs.

\section{CONCLUSION}

Delirium is increasingly associated with negative clinical outcomes, and recent guidelines ${ }^{8}$ (published after our study was performed) have highlighted the importance of appropriate screening and modification of risk factors. This multicentre study identified infrequent use of delirium screening tools in Canadian ICUs. Antipsychotics were prescribed frequently, and patterns of use were variable. We therefore believe there is an opportunity to improve delirium screening and management of mechanically ventilated patients in Canadian ICUs.

\section{References}

1. Delirium, dementia and amnestic and other cognitive disorders. In: Diagnostic and statistical manual of mental disorders. 4th ed. Washington (DC): American Psychiatric Association; 1994. p. 123-33.
2. Ely EW, Gautam S, Margolin R, Francis J, May L, Speroff T, et al. The impact of delirium in the intensive care unit on hospital length of stay. Intensive Care Med. 2001;27(12):1892-900.

3. Ely EW, Shintani A, Truman B, Speroff T, Gordon SM, Harrell FE Jr, et al. Delirium as a predictor of mortality in mechanically ventilated patients in the intensive care unit. JAMA. 2004:291(14):1753-62.

4. Girard TD, Jackson JC, Pandharipande PP, Pun BT, Thompson JL, Shintani AK, et al. Delirium as a predictor of long-term cognitive impairment in survivors of critical illness. Crit Care Med. 2010;38(7):1513-20.

5. Milbrandt EB, Deppen S, Harrison PL, Shintani AK, Speroff T, Stiles RA, et al. Costs associated with delirium in mechanically ventilated patients. Crit Care Med. 2004;32(4):955-62.

6. Klein Klouwenberg PMC, Zaal IJ, Spitoni C, Ong DSY, van der Kooi AW, Bonten MJM, et al. The attributable mortality of delirium in critically ill patients: prospective cohort study. BMJ. 2014;349:g6652.

7. Jacobi J, Fraser GL, Coursin DB, Riker RR, Fontaine D, Wittbrodt ET, et al. Clinical practice guidelines for the sustained use of sedatives and analgesics in the critically ill adult. Crit Care Med. 2002;30(1):119-41.

8. Barr J, Fraser GL, Puntillo K, Ely EW, Gelinas C, Dasta JF, et al. Clinical practice guidelines for the management of pain, agitation, and delirium in adult patients in the intensive care unit. Crit Care Med. 2013;41(1):263-306.

9. Ely EW, Inouye SK, Bernard GR, Gordon S, Francis J, May L, et al. Delirium in mechanically ventilated patients: validity and reliability of the Confusion Assessment Method for the Intensive Care Unit (CAM-ICU). JAMA. 2001; 286(21):2703-10.

10. Bergeron N, Dubois MJ, Dumont M, Dial S, Skrobik Y. Intensive care delirium screening checklist: evaluation of a new screening tool. Intensive Care Med. 2001;27(5):859-64.

11. Girard TD, Pandharipande PP, Ely EW. Delirium in the intensive care unit. Crit Care. 2008;12 Suppl 3:S3.

12. Patel RP, Gambrell M, Speroff T, Scott TA, Pun BT, Okahashi J, et al. Delirium and sedation in the intensive care unit: survey of behaviors and attitudes of 1384 healthcare professionals. Crit Care Med. 2009;37(3):825-32.

13. Swan JT, Fitousis K, Hall JB, Todd SR, Turner KL. Antipsychotic use and diagnosis of delirium in the intensive care unit. Crit Care. 2012;16(3):R84.

14. Mehta S, Burry L, Fischer S, Martinez-Motta JC, Hallett D, Bowman D, et al. Canadian survey of the use of sedatives, analgesics, and neuromuscular blocking agents in critically ill patients. Crit Care Med. 2006;34(2):374-80.

15. Gill KV, Voils SA, Chenault GA, Brophy GM. Perceived versus actual sedation practices in adult intensive care unit patients receiving mechanical ventilation. Ann Pharmacother. 2012;46(10):1331-9.

16. Elliott D, Aitken LM, Bucknall TK, Seppelt IM, Webb SA, Weisbrodt L, et al. Patient comfort in the intensive care unit: a multicentre, binational point prevalence study of analgesia, sedation and delirium management. Crit Care Resusc. 2013;15(3):213-9.

17. Burry LD, Williamson DR, Perreault MM, Rose L, Cook DJ, Ferguson ND, et al. Analgesic, sedative, antipsychotic, and neuromuscular blocker use in Canadian intensive care units: a prospective, multicentre, observational study. Can J Anaesth. 2014;61(7):619-30.

18. Luk E, Sneyers B, Rose L, Perreault MM, Williamson DR, Mehta S, et al. Predictors of physical restraint use in Canadian intensive care units. Crit Care. 2014;18(2):R46.

19. Dubois MJ, Bergeron N, Dumont M, Dial S, Skrobik Y. Delirium in an intensive care unit: a study of risk factors. Intensive Care Med. 2001; 27(8):1297-304.

20. Ouimet S, Kavanagh BP, Gottfried SB, Skrobik Y. Incidence, risk factors and consequences of ICU delirium. Intensive Care Med. 2007;33(1):66-73.

21. Van Rompaey B, Elseviers MM, Schuurmans MJ, Shortridge-Baggett LM, Truijen S, Bossaert L. Risk factors for delirium in intensive care patients: a prospective cohort study. Crit Care. 2009;13(3):R77.

22. Riker RR, Picard JT, Fraser GL. Prospective evaluation of the SedationAgitation Scale for adult critically ill patients. Crit Care Med. 1999;27(7):1325-29.

23. Harrell FE Jr. Regression modeling strategies with applications to linear models, logistic regression and survival analysis. New York (NY): Springer; 2001.

24. Neelon VJ, Champagne MT, Carlson JR, Funk SG. The NEECHAM confusion scale: construction, validation, and clinical testing. Nurs Res. 1996; 45(6):324-30 
25. Girard TD, Pandharipande PP, Carson SS, Schmidt GA, Wright PE, Canonico AE, et al. Feasibility, efficacy, and safety of antipsychotics for intensive care unit delirium: the MIND randomized, placebo-controlled trial. Crit Care Med. 2010;38(2):428-37.

26. Page VJ, Ely WE, Gates S, Zhao XB, Alce T, Shintani A, et al. Effect of intravenous haloperidol on the duration of delirium and coma in critically ill patients (Hope-ICU): a randomised, double-blind, placebo-controlled trial. Lancet Respir Med. 2013;1(7):515-23.

27. Devlin JW, Roberts RJ, Fong JJ, Skrobik Y, Riker RR, Hill NS, et al. Efficacy and safety of quetiapine in critically ill patients with delirium: a prospective, multicenter, randomized, double-blind, placebo-controlled pilot study. Crit Care Med. 2010;38(2):419-27.

28. Shehabi Y, Botha JA, Boyle MS, Ernest D, Freebairn RC, Jenkins IR, et al. Sedation and delirium in the intensive care unit: an Australian and New Zealand perspective. Anaesth Intensive Care. 2008;36(4):570-8.

29. Salluh JI, Dal-Pizzol F, Mello PV, Friedman G, Silva E, Teles JM, et al. Delirium recognition and sedation practices in critically ill patients: a survey on the attitudes of 1015 Brazilian critical care physicians. J Crit Care. 2009;24(4):556-62.

30. Jasiak KD, Middleton EA, Camamo JM, Erstad BL, Snyder LS, Huckleberry YC. Evaluation of discontinuation of atypical antipsychotics prescribed for ICU delirium. J Pharm Pract. 2013;26(3):253-6.

Zoé Thiboutot, BPharm, MSc, is a Pharmacist with the Centre hospitalier de l'Université de Montréal, Montréal, Quebec.

Marc M Perreault, BPharm, MSc, PharmD, is a Pharmacist with the McGill University Health Centre and a Full Clinical Professor with the Université de Montréal, Montréal, Quebec. He is also an Associate Editor with the CJHP.

David R Williamson, BPharm, MSc, PhD, is a Pharmacist with the Hôpital du Sacré-Coeur de Montréal and a Full Clinical Professor with the Université de Montréal, Montréal, Quebec.
Louise Rose, BN, MN, PhD, RN, is TD Nursing Professor of Critical Care Research with Sunnybrook Health Sciences Centre and an Associate Professor with the Lawrence S Bloomberg Faculty of Nursing, University of Toronto, Toronto, Ontario.

Sangeeta Mehta, MD, FRCPC, is an Intensivist with the Department of Medicine, Mount Sinai Hospital, and a Professor with the Faculty of Medicine, University of Toronto, Toronto, Ontario.

Melanie D Guenette, HBSC, MSc, is a Research Coordinator with the Department of Pharmacy, Mount Sinai Hospital, Toronto, Ontario.

Deborah Cook, MD, MSc, is an Intensivist with St Joseph's Healthcare and a Professor with the Departments of Medicine and of Clinical Epidemiology and Biostatistics, McMaster University, Hamilton, Ontario.

Lisa Burry, BScPharm, PharmD, is a Clinical Pharmacy Specialist with Mount Sinai Hospital and a Clinician Scientist with the Leslie Dan Faculty of Pharmacy, University of Toronto, Toronto, Ontario.

Competing interests: None declared.

\section{Address correspondence to:}

Dr Lisa Burry

Department of Pharmacy, Room 18-377

Mount Sinai Hospital

600 University Avenue

Toronto ON M5G 1X5

e-mail: Iburry@mtsinai.on.ca

Funding: This study was funded by Physicians' Services Incorporated Foundation and the Department of Pharmacy, Centre hospitalier de I'Université de Montréal.

Acknowledgements: The authors would like to thank Alex Kiss, PhD, for statistical support, and Stephanie C Lapinsky, BSc, for assistance with data entry.

\section{ON THE FRONT COVER}

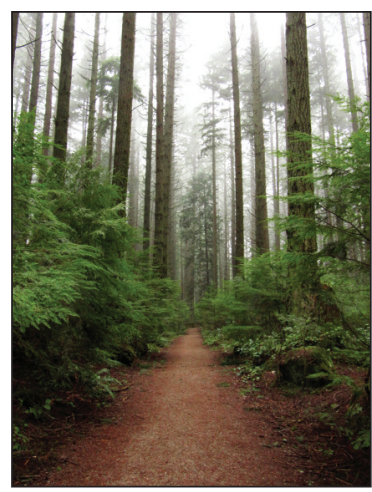

Pacific Spirit Regional Park

\section{Vancouver, British Columbia}

The cover photograph depicts new-growth forest at Pacific Spirit Regional Park in Vancouver, British Columbia, next to the campus of the University of British Columbia (UBC). Emma Attfield took the picture, and her brother, Benton Attfield, did some colour enhancement of the resulting digital image. At the time, they were both fourth-year students in the UBC Faculty of Pharmaceutical Sciences. Emma is now a Pharmacy Resident at Kelowna General Hospital, Interior Health (Kelowna, British Columbia), and Benton is now a Clinical Pharmacist with St Peter's Hospital, Hamilton Health Sciences (Hamilton, Ontario).

The CJHP would be pleased to consider photographs featuring Canadian scenery taken by CSHP members for use on the front cover of the journal. If you would like to submit a photograph, please send an electronic copy (minimum resolution $300 \mathrm{dpi}$ ) to cjhpedit@cshp.ca. 